\title{
Demographic Profile and Extra Intestinal Manifestations of Ulcerative Colitis in Nepalese population: Study from TUTH a Tertiary Care Centre, Kathmandu, Nepal.
}

\author{
Shaneel Harsh*, Ananya Adhikari, Parmatma Parazuli, Rahul Pathak, Prem K. Khadga, Sashi Sharma
}

Department of Gastroenterology, Tribhuvan University Teaching Hospital, Maharajgunj, Kathmandu

\section{DOI Name \\ http://dx.doi.org/10.3126/jaim.v4i1.14173}

\section{Keywords}

Endothelial dysfunction, high sensitive C-reactive protein, soluble E-selectin, Inflammatory marker

\section{Citation}

Shaneel Harsh, Ananya Adhikari, Parmatma Parazuli, Rahul Pathak, Prem K. Khadga, Sashi Sharma. Demographic Profile and Extra Intestinal Manifestations of Ulcerative Colitis in Nepalese population: Study from TUTH a Tertiary Care Centre, Kathmandu, Nepal. Journal of Advances in Internal Medicine 2015;04(01):1-5.

\begin{abstract}
Background and Aims: This study aims at determining the demographic profile and extra-intestinal manifestations of Ulcerative Colitis in the Nepalese patients treated in a tertiary referral centre.

Methods: The study was conducted between February 1, 2014 and January 31, 2015 at the Department of Gastroenterology Tribhuvan University Teaching Hospital, Kathmandu, Nepal. The clinical and epidemiologic data from patients diagnosed to have Ulcerative Colitis were obtained and analysed.

Results: During the study period, 60 patients were identified as having ulcerative colitis. Mean age at diagnosis was 34.6 years. The mean duration of illness at diagnosis was 3.7 years. Extra-intestinal manifestations (EIM) were seen in $16.6 \%$ of the patients. Sacroilitis was the most common EIM seen in $11.6 \%$ followed by peripheral arthritis in $6.6 \%$ of the patients. Episcleritis, Primary Sclerosing Cholangitis and Erythema Nodosum were seen only in $1.6 \%$ each. At the time of presentation, 38.3\% $(n=23)$ had proctosigmoiditis, $50 \%$ $(n=30)$ left sided colitis and pancolitis was seen in $11.7 \%(n=7) .53 .3 \%$ patients had mild disease, $38.3 \%$ and $8.3 \%$ presented with moderate to severe disease respectively.

Conclusion: Peak age of onset for UC in the study was third and fourth decade which was similar to the various studies from Asia and West. Musculoskeletal manifestation was the most common extra- intestinal manifestation of UC in Nepalese population. As Nepal is perhaps the most diverse country in terms of ethnicity, cultural variation, socio-economic status and health care facilities, a comprehensive nationwide data bank involving ethnicity and geographical variation is needed for a better definition of the disease characteristics.
\end{abstract}

\section{INTRODUCTION}

Ulcerative colitis is a chronic inflammatory bowel disease of unknown etiology. It is a worldwide disorder with significant geographical heterogeneity, the highest prevalence rates having been reported from Northern and Western Europe and North America. ${ }^{1}$ A significant North-South gradient has also been observed in the prevalence of the disease, with rates being higher in Northern countries. ${ }^{2,3}$ Ulcerative colitis has been viewed from various studies in the past as being rare in developing countries of the world, including those from
Asia. ${ }^{4}$ However, recent epidemiological experience is changing these premises. The disease is beginning to make itself known in populations where it had never been seen before, such as the the Chinese in Singapore, and the Arabs in Kuwait. ${ }^{5}$ An increased incidence is also being reported from the previously low incidence areas of Australia and Eastern Europe. 6, 7

\footnotetext{
* Corresponding author

Dr Shaneel Harsh

Department of Gastroenterology, Tribhuvan University Teaching Hospital

Maharajgunj, Kathmandu

drshaneelharsh@gmail.com
} 
Moreover high prevalence and incidence has also been found in northern India. ${ }^{8}$

Ulcerative colitis as a systemic disorder often involving other organs. Involvement of organs other than the intestine is called as Extra Intestinal Manifestations and often complicates its management and, is a significant source of morbidity and mortality. ${ }^{10}$ The frequency and temporal relation of these manifestations have led to general acceptance that these intestinal problems are systemic disorders, with wide spectrum of extra intestinal manifestation which includes pyoderma, gangrenosum, erythema nodosum, uveitis, episcleritis, cholestatic liver disease, hemolytic anemia, arthritis, and pulmonary disease of various types. ${ }^{9}, 11,12,13$ Various studies reported from various parts of world showed differences amongst the demographic and clinical characterstics of patients with Ulcerative Colitis.

Despite the rising incidence and prevalence of this condition in Asian countries, the data regarding this disease in Nepalese patients are relatively few or none and may be different from the Western counterparts. In view of this, to determine demographic profile and extra intestinal manifestations of Ulcerative Colitis in Nepalese population, we conducted this study in TUTH, a tertiary care centre in Nepal,

\section{MATERIALS AND METHODS}

All patients with Ulcerative Colitis presenting to the Outpatient department or admitted in the ward of Department of Gastroenterology, Tribhuvan University Teaching Hospital, Kathmandu in the study period of one year (February $1^{\text {st }} 2014$ to January $1^{\text {st }} 2015$ ) were included in the study.

A definite diagnosis of UC was made in those who met all 3 of the criteria used for determining UC:

(1) A typical history of diarrhoea or blood and pus in the stool, or both, for longer than 4 weeks;

(2) A typical sigmoidoscopic or colonoscopic picture with diffusely granular, friable, or ulcerated mucosa without rectal sparing or skip lesions of characteristic continuous ulcerated mucosa; and

(3) Characteristic histopathological signs of inflammation on biopsy. ${ }^{14,15,16,17}$

Following clinical data were retrieved from each patient:
(i) $\operatorname{sex}$
(ii) age at diagnosis
(iii) duration of disease
(iv) presenting symptoms

\section{(v) family history of UC}

(vi) extent of colitis(vii) severity of disease at diagnosis (viii) extraintestinal manifestations.

Extent of colitis was assessed by the first-time colonoscopy and was classified as ulcerative proctitis (E1), left-sided UC (E2), and extensive UC (E3) according to the Montreal classification of IBD. ${ }^{18}$

The disease severity was classified clinically according to Truelove and Witts criteria as mild, moderate, severe and fulminant colitis. ${ }^{19}$

\section{STATISTICS}

All the data were collected as per standard prespecified Performa and all statistical analysis were performed with Statistical Package for Social Sciences version 20.0 software.

\section{RESULTS}

A total of 60 patients presented to the Department of Gastroenterology during the study period. All of them were Nepalese. 31 patients were male and 29 patients were female. ( $\mathrm{M}: \mathrm{F}=1.06: 1$ ). The mean age at diagnosis was 34.6 years with standard deviation of 12.7 years.

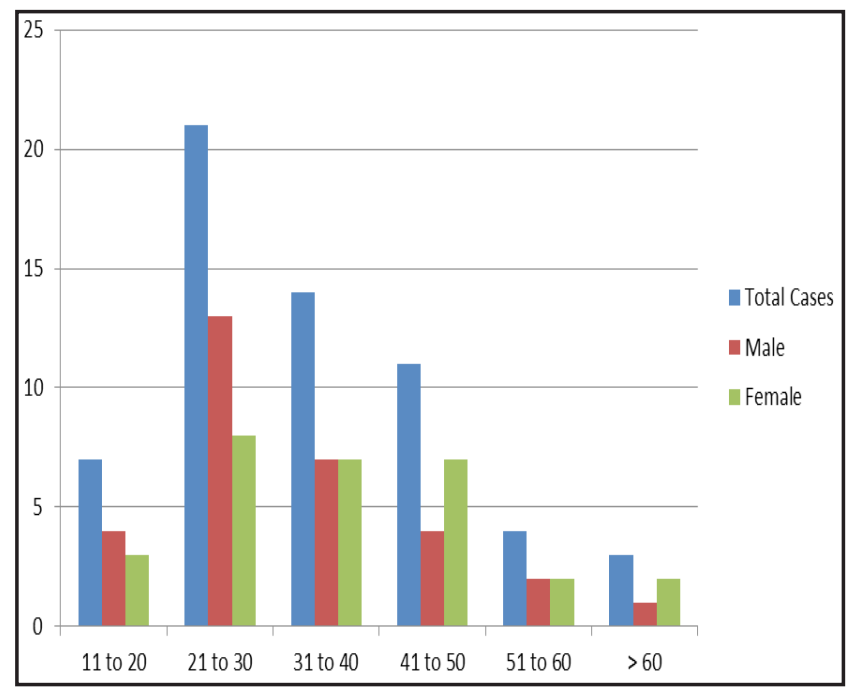

Figure 1: Demographic distribution of Patients

The mean duration of illness at diagnosis was 3.7 years with standard deviation of 4.87 years

At presentation $38.3 \%(\mathrm{n}=23)$ had proctosigmoiditis, $50 \%$ $(n=30)$ had left sided colitis and pancolitis was seen in $11.7 \%$ $(n=7)$.

53.3\% patients had mild disease, $38.3 \%$ and $8.3 \%$ presented with moderate to severe disease respectively. $3.33 \%(n=2)$ had to undergo surgical therapeutic colectomy. 


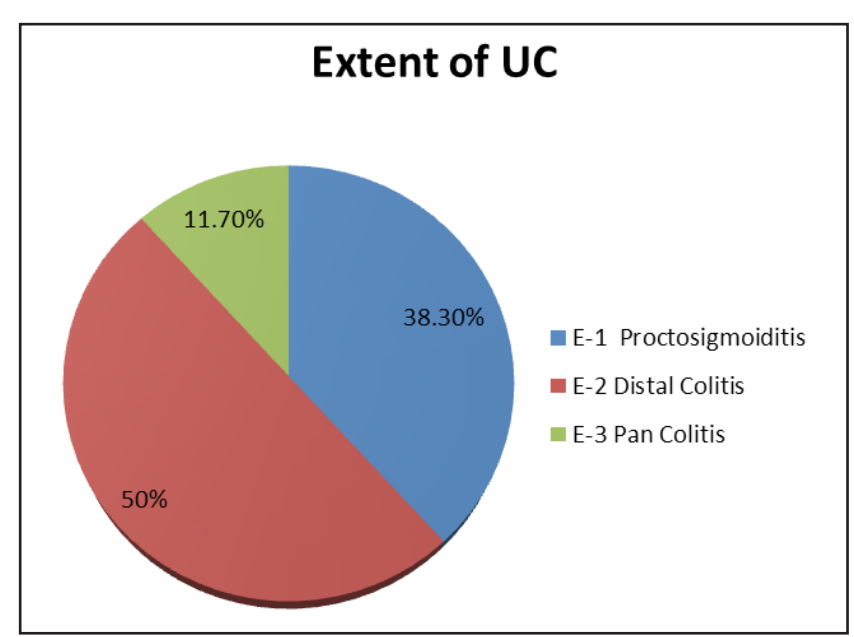

Figure 2: Extent of disease

Majority (86.7\%), of the patients had clinical course of Chronic Intermittent type. Other two types were present with Chronic Continuous in $10 \%$ ) and Acute Fulminant in $3.3 \%$ of cases.

Table 1: Extraintestinal manifestations

\begin{tabular}{ll}
\hline Extraintestinal manifestations & Percentage (\%) \\
\hline Sacroilitis & 11.6 \\
Peripheral Arthitis & 6.66 \\
Episcleritis & 1.66 \\
Erythema Nodosum & 1.66 \\
Primary Sclerosing Cholangitis & 1.66 \\
\hline
\end{tabular}

Extra intestinal manifestations were seen in $16.6 \%(n=10)$ of all cases Musculoskeletal symptoms were present in $13.3 \%(n=8)$; Sacroilitis being the most common EIM symptom manifesting in $11.6 \%(n=7) .43 \%$ of patients with sacroilitis also had peripheral arthritis Peripheral arthritis alone was also seen in $1.7 \%(n=1)$.Episcleritis was present in $1.66 \%(n=1)$.Primary Sclerosing Cholangitis and Erythema Nodosum was only seen in $1.6 \%(n=1)$ each.

\section{DISCUSSION}

Ulcerative colitis should no longer be considered as a disease of Western countries. A number of studies showed that there is a rising prevalence of this disease across Asian countries. ${ }^{20,}$ 21,22

The present study identified important demographic information. This study reports peak age of onset in $3^{\text {rd }}$ and $4^{\text {th }}$ decade which is similar to reports from asia and western countries. Studies of western world even reports a second smaller peak in incidence seen typically in the 6th to 7th decade which has not been seen in this study and this finding is consistent with the studies from Asia. . ${ }^{23,24,25}$

This study shows that UC is distributed equally between males and females ( $M: F=1.06: 1)$.The finding is similar to reports from east and west parts of the world that states that the gender distribution is almost equal for UC.

A family history of UC was previously noted to be uncommon among Asian studies, with a frequency ranging from $1.6 \%$ to $4.5 \%$, which is considerably lower than the reported rates from Western series ( $8 \%-14 \%) \cdot 4,20,23$ This study also shows family aggregation in $1.6 \%$ which is very much similar to the Chinese studies. ${ }^{26,27}$

Our study showed majority of disease is left sided colitis $50 \%$ and $11.7 \%$ of patient diagnosed as Pancoliitis whereas reports from western countries shows $27-62.7 \%$ of patients suffering from pancolitis. ${ }^{21,22}$ Similar findings were shown in many Asian reports mainly from Iran and China. ${ }^{27,24}$

Another significant result of this study is the delay in the diagnosis of Ulcerative Colitis in the Nepalese population. As per Turkish study 1.2 years duration of disease was considered to be late. ${ }^{28}$ Our study reports 3.7 years duration of disease at diagnosis which is significantly delayed. There may be several explanations for this finding. First, non-specific initial symptoms of Ulcerative Colitis might be underrated by the patients. Secondly, higher prevalence of infectious diseases and common disorders such as haemorrhoids may have resulted in misdiagnosis of the disease. Thirdly, the negative attitudes of patients towards endoscopic procedures and lack of availability of the diagnostic facilities might also have contributed in the delay of the diagnosis.

Ulcerative colitis is a systemic disorder that often involves other organs. This involvement of other organs than intestine is known as Extra Intestinal Manifestations and often complicates its management and is a significant source of morbidity and mortality. ${ }^{10}$

This study shows Extra-intestinal manifestations were present in $16.6 \%$, which was far less than that reported from the Western countries reports of $25-47 \% .^{12,13}$ Indian studies also reports EIM from $4 \%$ to recent reports of $50.6 \%$ from ISG task force.$^{29,30,31}$ Studies from China and Taiwan however have reported low EIM of 5-6\%. ${ }^{32}$

Musculoskeletal system (13.3\%) is the most common Extraintestinal manifestation in this study. Reported prevalence of musculoskeletal Extra-intestinal manifestations in Inflammatory Bowel Disease patients ranges from 9\% to $47 \% .{ }^{27,33,6}$ Sacroilitis was seen in $11.6 \%$ patients, This findings is supported by the reports showing incidence of sacroilitis is up to $52 \%$ of the patients in UC. ${ }^{29,30,34}$ A study from Italy reports $50 \%$ of UC patients have characteristic inflammatory low back pain in the absence of radiological findings suggestive of sacroileitis ${ }^{35} .43 \%$ of the patients diagnosed as sacroilitis in this study also had peripheral arthritis. Peripheral arthritis 
alone was seen only in $1.6 \%$.

Specifically for UC, the rates of Pyoderma Gangrenosum reported has been reported as high as $4 \%-5 \%$ and as low as $0.5 \%$ and $0.8 \%$ in various studies. ${ }^{12,36,23}$ Rates for erythema nodosum have been reported in the range $0.9 \%$ to $4 \% .{ }^{12,37}$ This study does not report any case of Pyoderma Gangrenosum. Erythema Nodosum was seen on a lower side of $1.6 \%$ which is consistent to many western reports as well.

Ophthalmologic complication has been reported as 1 to $4 \%$ in various studies. ${ }^{12,33}$ This study does not report any case of uveitis and episcleritis was seen in only $1.7 \%$ which is very much consistent with the study from main land China. ${ }^{27}$

Primary Sclerosing Cholangitis is a disorder of both intrahepatic and extrahepatic bile ducts. The prevalence of Primary Sclerosing Cholangitis in Ulcerative Colitis has ranged from $2.4 \%$ to $7.4 \%$ amongst various studies $.38,39$ Previously, only scant reports of PSC in UC patients have been published, but no prevalence of such a condition has been reported in the Asia Pacifc. Our findings regarding PSC with a prevalence of $1.7 \%$ are consistent with the study in Taiwan but comparatively less than the reports from Iran and Western Europe.

\section{CONCLUSION:}

Peak age of onset for Ulcerative Colitis in this study was found to be in the $3^{\text {rd }} \& 4^{\text {th }}$ decade which was similar to the various studies reported from Asia and Western countries. There was an absence of second peak in this study which is consistent with the studies from Asia but differs from the reports of Western world.

Extra -intestinal manifestations were low in our study as compared to studies reported from western world and few recent studies from India. Musculoskeletal manifestations were the most common Extra- intestinal manifestation of UC in the Nepalese population.

Delay in the diagnosis of Ulcerative Colitis was seen in the Nepalese population which might be related to the lack of awareness and health care resources.

As Nepal is perhaps the most diverse country in terms of ethnicity, cultural variation, socio-economic status and health care facilities, a comprehensive nationwide data bank involving ethnicity and geographical variation is needed for a better definition of the disease characteristics.

Incidence and prevalence of Ulcerative Colitis. Gut 2003 52: $1587-1590$

9. Levine, JB, Lukawski-Trubish, D (1995) Extraintestinal considerations in inflammatory bowel disease. Gastroenterol Clin North Am24,633-646.

10. Kirsner JB,Inflammatory bowel disease:Consideration of etiology and pathogenesis.Am J Gatroenterology $65: 253,1978$

11. Bargen JA: Complications and sequelae of chronic ulcerative colitis.Ann Intern Med 1929;3:335-52

12. Greenstein AJ,JanowitzHD,Sacchar DB:The Extra intestinal complications of Crohn's and ulcerative colitis:A study of 700 patients.Medicine 55:401,1976.

13. Rankin GB,Watts HD,Melnyk CS:National Cooperative Crohn's Disease Study (NCCDS)Extra intestinal manifestations and perianal complications .Gastroenterology 77:914,1979.

14. Yang SK, Hong WS, Min $\mathrm{YI}$, et al. Incidence and prevalence of ulcerative colitis in the Songpa-Kangdong District, Seoul, Korea, 1986-1997. J Gastroenterol Hepatol. 2000;15:1037-1042.

15. Tysk C, Jarnerot G. Ulcerative proctocolitis in Orebro,

8. A Sood, V Midha, N Sood, et al.Punjab, North India. 
Sweden. A retrospective epidemiologic study, 19631987. Scand J Gastroenterol. 1992;27:945-950.

16. Garland CF, Lilienfeld AM, Mendeloff Al, et al. Incidence rates of ulcerative colitis and Crohn's disease in fifteen areas of the United States. Gastroenterology. 1981;81:1115-1124.

17. Binder $\mathrm{V}$, Both $\mathrm{H}$, Hansen $\mathrm{PK}$, et al. Incidence and prevalence of ulcerative colitis and Crohn's disease in the County of Copenhagen, 1962 to 1978. Gastroenterology. 1982;83:563-568

18. Satsangi J, Silverberg MS, Vermeire S, Colombel JF. The Montreal classification of inflammatory bowel disease: controversies, consensus and implications. Gut 2006; 55: 749-53.

19. Truelove SC, Witts LJ. Cortisone in ulcerative colitis. Final report on a therapeutic trial. BMJ 1955; 2: 1041-5.

20. Yang $H$, McElree $C$, Roth $M P$, et al. Familial empirical risks,for inflammatory bowel disease: Differences between Jews and non-Jews. Gut 1993;34:517-24.

21. Nguyen GC, Torres EA, Regueiro M, et al. Inflammatory bowel disease characteristics among African Americans,Hispanics, and non-Hispanic Whites: Characterization of a large North American cohort. Am J Gastroenterol 2006;101:1012-23.

22. Farmer RG, Easley KA, Rankin GB. Clinical patterns, natural history, and progression of ulcerative colitis. A long-term follow-up of 1116 patients. Dig Dis Sci 1993;38:1137-46

23. Monsen U, Brostrom O, Nordenvall B, et al. Prevalence of inflammatory bowel disease among relatives of patients with ulcerative colitis. Scand J Gastroenterol $1987 ; 22: 214$

24. Epidemiological, Demographic, and Colonic Extension of Ulcerative Colitis in Iran: A Systematic Review Ali Akbar Shayesteh ,Mehdi Saberifirozi, Shifteh Abedian, and Vahid Sebghatolahi Middle East J Dig Dis. 2013 Jan; 5(1): 29-36.

25. Frequency and Risk Factors for Extraintestinal Manifestations in the Swiss Inflammatory Bowel Disease Cohort Stephan R Vavricka MD Lionel Brun,Pierluigi Ballabeni, Valérie Pittet, Bettina Mareike Prinz Vavricka $M D$, Jonas Zeitz MD, Gerhard Rogler MD and Alain M Schoepfer MD and the Swiss IBD Cohort Study Group Am J Gastroenterol 2011; 106:110-119
26. YuFang WANG, Hu ZHANG \& Qin OUYANG Clinical manifestations of inflammatory bowel disease:East and West differences. Journal of Digestive Diseases 2007; 8; 121-127.

27. Jiang XL, Cui HF. An analysis of 10218 ulcerative colitis cases in China. World J. Gastroenterol. 2002; 8: 158-61.

28. Clinical Features of Ulcerative Colitis and Crohn's Diseasein Turkey Yasemin Ozin, Mesut Zeki Yalin Kilic, Isilay Nadir, Basak Cakal, Selcuk Disibeyaz, Mehmet Arhan, Ulku Dagli, Bilge Tunc, Aysel Ulker, Burhan Sahin. J Gastrointestin Liver Dis June 2009 Vol.18 No 2, 157-162

29. Tandon BN, Mathur AK,Mohapatra LN et al, A study of prevalence and clinical pattern of nonspecific ulcerative colitis in Northern India.Gut 1965;6(5):448-53.

30. Chuttani HK,Nigam SP,Sama SK etal .Ulcerative colitisin the tropics.British Medical Journal 1967;4:204-07.

31. Indian J Gastroenterol (November-December 2012) 31(6):307-323.

32. Jiang L, Xia B, Li J et al. Retrospective survey of 452 patients with inflammatory bowel disease in Wuhan city, central China. Inflamm. Bowel Dis. 2006; 12: 212-17.

33. Bernstein, C. N., Blanchard, J. F., Rawsthorne, P. \& $\mathrm{Yu}, \mathrm{N}$. The prevalence of extraintestinal diseases in inflammatory bowel disease: a population-based study. Am. J. Gastroenterol. 96, 1116-1122 (2001).

34. Rao SS,HoldsworthCD, Read NW.Symptoms and stool pattern in patients with ulcerative colitis. Gut $1988 ; 29: 342$

35. Extraintestinal manifestations in inflammatory bowel disease Silvio Danese, Stefano Semeraro, Alfredo Papa, Italia Roberto, Franco Scaldaferri, Giuseppe Fedeli, Giovanni Gasbarrini,Antonio Gasbarrini World J Gastroenterol 2005;11(46):7227-7236

36. Basler RSW. Ulcerative colitis and the skin. Med Clin North Am 1980;64:941

37. Orchard T. Extraintestinal complications of inflammatory bowel disease. Curr Gastroenterol Rep 2003; 5: 512-517.

38. Danese S, Semerano S, Papa A, et al. Extraintestinal manifestations in inflammatory bowel disease. World J Gastroenterol 2005; 11: 7227-7236

39. Y.M. Lee andM.M. Kaplan, "Primary sclerosing cholangitis," New England Journal of Medicine, vol. 332, no. 14 , pp. 924-933,1995. 